\title{
Evaluation of Several Tests in Screening for Chloroquine Maculopathy
}

\author{
S. A. BISHARA and N. MATAMOROS \\ Jerusalem, Israel
}

\begin{abstract}
Summary
Patients receiving antimalarial therapy, specifically hydroxychloroquine, for different periods were evaluated using contrast sensitivity test (CST) and results were compared with those of electro-retinography (EOG), and pattern visual evokea potential (PVEP), and a matching age control group. The results indicated CST to be most sensitive of the evaluated techniques particularly in patients under 40 years old. In $44.4 \%$ of the cases CST revealed macular dysfunction of which the other two methods of examination were not capable. Our findings suggest the CST is a reliable and practical method which could be used as an additional screening test for chloroquine maculopathy.
\end{abstract}

Clinicians are making every possible effort to achieve early diagnosis of chloroquine retinopathy which is particularly important in the early stages of this condition, when there is still a possibility for reversal.

With the recently increasing use of chloroquine and chloroquine derivatives in the treatment of connective tissue diseases, ${ }^{1,2}$ early diagnosis of chloroquine maculopathy has become imperative. In an attempt to achieve this, many tests have been proposed, including visual fields, colour vision, Amsler grid, and fluorescein angiography. ${ }^{3,4,5,6,7}$

Electro-oculography (EOG) is one of these tests, and has been reported to be a sensitive test, ${ }^{8}$ although this is still controversial. ${ }^{9}$ The test is still used in several centres for the detection of functional disturbances induced by the storage of chloroquines in the retinal pigment epithelium. ${ }^{10}$

The spatial contrast sensitivity test (CST), using sinusoidal gratings, is frequently recommended as a sensitive means of detecting and evaluating several macular diseases. ${ }^{11,12}$ The pattern visual evoked potential (PVEP) has also been suggested for testing macular disorders. ${ }^{13,14}$

We report the results and relative sensitivity of the CST, EOG and PVEP tests in revealing early chloroquine maculopathy in 27 patients treated for long periods with hydroxychloroquine, which is a less toxic derivative of chloroquine.

\section{Patients and Methods}

Twenty-seven patients, ranging in age from 21 to 80 years and suffering from rheumatoid arthritis, systemic lupus erythematosus, familial Mediterranean fever and other collagen diseases, were examined (Table I). The subjects were treated with hydroxychloroquine at a dosage of $200 \mathrm{mg}$./day for periods ranging from 1 to 9 years.

A second, randomly chosen, group of 28 patients, of similar age, served as a control (Table II). Some of these subjects suffered 
Table I Results of various evaluations and duration of treatment in patients under antimalarial drugs

\begin{tabular}{|c|c|c|c|c|c|c|c|c|}
\hline \multirow[b]{2}{*}{ Age } & \multirow{2}{*}{$\begin{array}{l}\text { Years of } \\
\text { treatment }\end{array}$} & \multirow{2}{*}{$\begin{array}{c}\text { corrected } \\
\qquad E\end{array}$} & \multirow{2}{*}{$\begin{array}{l}V . A . \\
L E\end{array}$} & \multirow{2}{*}{$\begin{array}{l}\text { E.O.G. } \\
\quad R E\end{array}$} & \multirow{2}{*}{$\begin{array}{l}\text { Arden Ind. } \\
\quad L E\end{array}$} & \multicolumn{2}{|c|}{ C.S.T. } & \multirow{2}{*}{$\begin{array}{l}\text { P.V.E.P } \\
\text { BE }\end{array}$} \\
\hline & & & & & & $R E$ & $L E$ & \\
\hline \multicolumn{9}{|c|}{ Subgroup A } \\
\hline 21 & 2 & $6 / 6$ & $6 / 6$ & 270 & 290 & 46 & 46 & normal \\
\hline 21 & 2 & $6 / 6$ & $6 / 6$ & 340 & 340 & 55 & 55 &.. \\
\hline 21 & 2 & $6 / 6$ & $6 / 6$ & 290 & 300 & 64 & $67+$ & .. \\
\hline 34 & 1.5 & $6 / 6$ & $6 / 6$ & 260 & 260 & 57 & 59 & .. \\
\hline 37 & 4 & $6 / 6$ & $6 / 6$ & 190 & 180 & 61 & 60 & .. \\
\hline 39 & 1.5 & $6 / 9$ & $6 / 9$ & 240 & 210 & 63 & $66+$ & .. \\
\hline 40 & 3 & $6 / 9$ & $6 / 9$ & 200 & 220 & 75 & $65+$ & Sub $\cdot$ \\
\hline \multicolumn{9}{|c|}{ Subnormal B } \\
\hline 41 & 3 & $6 / 9$ & $6 / 9$ & 290 & 280 & 53 & 56 & normal \\
\hline 41 & 7 & $6 / 6$ & $6 / 6$ & 320 & 350 & 51 & 54 &. \\
\hline 43 & 2 & $6 / 6$ & $6 / 6$ & 245 & 285 & 47 & 45 & .. \\
\hline 43 & 7 & $6 / 6$ & $6 / 6$ & 210 & 200 & 42 & 45 & .. \\
\hline 43 & 3 & $6 / 9$ & $6 / 9$ & 190 & 285 & 59 & 60 & .. \\
\hline 47 & 2 & $6 / 6$ & $6 / 6$ & 220 & 230 & 58 & 59 & .. \\
\hline 48 & 4 & $6 / 9$ & $6 / 9$ & 300 & 330 & 62 & 58 & .. \\
\hline 50 & 8 & $6 / 6$ & $6 / 6$ & 200 & 180 & 63 & $68+$ & .. \\
\hline 51 & 1 & $6 / 6$ & $6 / 6$ & 235 & 235 & 67 & $64+$ & .. \\
\hline 53 & 2 & $6 / 6$ & $6 / 6$ & 220 & 235 & 48 & 53 & .. \\
\hline 53 & 6 & $6 / 9$ & $6 / 12$ & 230 & 180 & 116 & $118+$ & .. \\
\hline 54 & 8 & $6 / 12$ & $6 / 12$ & 285 & 230 & 74 & $64+$ & .. \\
\hline 55 & 3 & $6 / 9$ & $6 / 9$ & 265 & 220 & 52 & 51 & .. \\
\hline 59 & 2 & $6 / 24$ & $6 / 7.5$ & 275 & 268 & 50 & 61 & .. \\
\hline 60 & 3 & $6 / 6$ & $6 / 6$ & 156 & $145+$ & 77 & $67+$ &.. \\
\hline 64 & 2 & $6 / 12$ & $6 / 9$ & 133 & $150+$ & 108 & $101+$ & .. \\
\hline 64 & 1 & $6 / 9$ & $6 / 9$ & 172 & $172+$ & 90 & $100+$ &.. \\
\hline 68 & 8 & $6 / 9$ & $6 / 9$ & 225 & 257 & 76 & $82+$ & Sub $\cdot$ \\
\hline 74 & 9 & $6 / 12$ & $6 / 12$ & 270 & 200 & 50 & 41 & normal \\
\hline 80 & 3 & $6 / 15$ & $6 / 12$ & 130 & $125+$ & 85 & $91+$ & .. \\
\hline
\end{tabular}

${ }^{*}$ Arden Ind.: Arden Index

† Subnormal or borderline values.

from dry eyes or various types of conjunctivitis, others were referred for a driving license test.

Each patient underwent a full ophthalmological examination, including visual acuity, ocular pressure, slit lamp biomicroscopy and direct and indirect fundus ophthalmoscopy, with emphasis on the appearance of the macula. Among the treated patients the average intraocular pressure ranged between 14 and $22 \mathrm{mmHg}$; the anterior segments were normal in all patients except for mild lenticular sclerosis seen in 4 patients, though their visual acuity was maintained at a $6 / 6-6 / 12$ level. The fundus findings were within normal limits in all these patients, but two patients showed fine pigmentary stippling of the macula and age-related peripheral pigmentary changes.

In the control group six patients aged 58 years and above had mild lenticular sclerosis which affected their visual acuity to the level of $6 / 12$.

Patients with media opacity, ocular or macular pathology or any other aetiology which affected their visual acuity, or appeared to affect the CST, were excluded from the study.

The CST was performed using AO contrast sensitivity test plates, with each plate at a test distance of about $57 \mathrm{~cm}$. from the eyes, producing a $2 \mathrm{c} /$ degree at plate 2 and up to $6.4 \mathrm{c} /$ degree at plate 7 . The test was conducted at the recommended illumination level of $100 \mathrm{ft}$. candles. After explanation and presentation of the demonstration plate (plate 1) to the patient, each plate was slowly drawn from the original box at a full plate exposure speed of 15 seconds. In elderly patients the plate exposures were repeated twice, in order to 
Table II Control Group Evaluations

\begin{tabular}{|c|c|c|c|c|c|c|c|}
\hline \multirow[b]{2}{*}{ Age } & \multirow{2}{*}{$\begin{array}{l}\text { Visual } \\
\quad R E\end{array}$} & \multirow{2}{*}{$\begin{array}{l}\text { acuity } \\
\text { LE }\end{array}$} & \multirow{2}{*}{$\begin{array}{l}\text { E.O.G. } \\
\quad R E\end{array}$} & \multirow{2}{*}{$\begin{array}{l}\text { Arden Ind.* } \\
\text { LE }\end{array}$} & \multicolumn{2}{|c|}{ C.S.T. } & \multirow{2}{*}{$\begin{array}{c}\text { P.V.E.P. } \\
B E\end{array}$} \\
\hline & & & & & $R E$ & $L E$ & \\
\hline \multicolumn{8}{|c|}{ Subgroup C } \\
\hline 20 & $6 / 6$ & $6 / 6$ & 210 & 210 & 54 & 54 & normal \\
\hline 20 & $6 / 6$ & $6 / 6$ & 220 & 215 & 59 & 58 &.. \\
\hline 21 & $6 / 7.5$ & $6 / 6$ & 230 & 200 & 53 & 44 &.$\cdot$ \\
\hline 23 & $6 / 6$ & $6 / 9$ & 195 & 200 & 59 & 54 & $\cdot$. \\
\hline 25 & $6 / 6$ & $6 / 6$ & 190 & 185 & 58 & 60 &.. \\
\hline 28 & $6 / 6$ & $6 / 6$ & 240 & 225 & 46 & 48 &.$\cdot$ \\
\hline 33 & $6 / 6$ & $6 / 6$ & 210 & 205 & 51 & 58 &.. \\
\hline 35 & $6 / 7.5$ & $6 / 7.5$ & 195 & 210 & 56 & 53 &.. \\
\hline 39 & $6 / 6$ & $6 / 6$ & 200 & 215 & 49 & 53 &.. \\
\hline 40 & $6 / 6$ & $6 / 6$ & 210 & 220 & 60 & 57 & $\cdot$. \\
\hline \multicolumn{8}{|c|}{ Subgroup D } \\
\hline 41 & $6 / 6$ & $6 / 6$ & 235 & 215 & 61 & 59 & $\cdot$. \\
\hline 41 & $6 / 6$ & $6 / 6$ & 190 & 205 & 55 & 53 &.$\cdot$ \\
\hline 45 & $6 / 6$ & $6 / 6$ & 210 & 220 & 56 & 56 &.. \\
\hline 47 & $6 / 6$ & $6 / 6$ & 185 & 195 & 62 & 61 &.$\cdot$ \\
\hline 48 & $6 / 6$ & $6 / 9$ & 190 & 200 & 40 & 45 &.. \\
\hline 50 & $6 / 9$ & $6 / 6$ & 225 & 215 & 49 & 51 & .. \\
\hline 53 & $6 / 6$ & $6 / 6$ & 230 & 245 & 55 & 59 & .. \\
\hline 55 & $6 / 6$ & $6 / 6$ & 215 & 220 & 61 & 62 &.. \\
\hline 58 & $6 / 7.5$ & $6 / 9$ & 230 & 240 & 54 & 57 &.. \\
\hline 58 & $6 / 6$ & $6 / 6$ & 200 & 195 & 68 & $71+$ & .. \\
\hline 60 & $6 / 12$ & $6 / 9$ & 223 & 215 & 50 & 52 &.. \\
\hline 63 & $6 / 7.5$ & $6 / 6$ & 194 & 195 & 53 & 56 &.. \\
\hline 66 & $6 / 6$ & $6 / 6$ & 205 & 207 & 61 & 58 &.. \\
\hline 71 & $6 / 12$ & $6 / 9$ & 170 & $175+$ & 75 & $80+$ &.. \\
\hline 73 & $6 / 9$ & $6 / 9$ & 185 & 180 & 57 & 59 &.. \\
\hline 75 & $6 / 9$ & $6 / 6$ & 195 & 205 & 57 & 56 &.. \\
\hline 78 & $6 / 12$ & $6 / 12$ & 175 & $168+$ & 84 & $79+$ &.. \\
\hline 80 & $6 / 9$ & $6 / 9$ & 215 & 210 & 63 & 60 &.. \\
\hline
\end{tabular}

* Arden In.: Arden Index

† Subnormal or borderline values.

avoid learning difficulty and to get a consistent performance of the test. The score was recorded as the patient's first response. The total score for all the plates was taken as the final result. A score of 62 or less was considered normal, that between 63-78 was considered suspect, and above that was definitely abnormal.

The EOG was performed by sitting patients at a distance of one metre from the screen, with two fixation points at a distance of $30^{\circ}$ one from the other. The electrodes were attached to the medial and lateral canthus of each eye, and an indifferent electrode was attached to the ear. At a given rhythm, the patient was instructed to alternate his fixation between the two points, for one minute at a time. The examination was performed in a dark room for 15 minutes and repeated in a lighted room for a further 15 minutes. The Arden index was calculated by dividing the light peak/dark trough. A score of 1.8 or $180 \%$ was considered the lower normal limit ( 0.2 or $20 \%$ is the standard deviation in our laboratory).

The PVEP was performed in a semi-sitting position, at a distance of $1.5 \mathrm{~m}$ from a TV checkerboard screen stimulator with $95 \%$ contrast and $1.88 \mathrm{c} / \mathrm{sec}$ frequency and $36 \mathrm{sec}$ check size. The electrodes were attached to the scalp with pentonate paste $5 \mathrm{~cm}$ above the $3 \mathrm{~cm}$ lateral to the inion on each hemisphere. The reference electrode was attached to the forehead and the indifferent electrode to the ear. Each eye was stimulated separately, and the unexamined eye was covered with a heavy black tissue and the patient's palm. The average response following 100 stimuli was 
recorded on a film, with $110 \mathrm{msec}$ considered the normal first major peak latency.

\section{Results}

As shown in Table I, the CST was normal in 15 of the 27 treated patients, suspect in seven patients, and definitely abnormal in five patients - all five having been on antimalarial treatment for periods ranging from one to eight years.

The EOG results were normal in 23 out of 27 patients. One patient showed a borderline Arden index (1.7), and the remaining three patients showed definitely pathologic Arden indices, two of these three patients had been on antimalarial medication for two years, and the third for three years. These four patients were also included in the five cases which showed a pathologic CST.

The PVEP was normal in 25 out of 27 patients. In two patients there was a latency of more than $110 \mathrm{msec}$. In these two cases the EOG was normal, but the CST was suspect. No pathology was discerned which could account for the abnormal PVEP.

In the control group (Table II), the CST was subnormal in two and borderline in one of the 28 cases. One of the 28 control patients was considered normal though the right eye scored 63, just above the upper limit (62), and the left eye was within the normal range at 60 . the EOG was subnormal in only two of the 28 controls. The PVEP was normal in all the controls.

When the two groups were subdivided, using 40 years as the separating age, the treated group was now divided into seven young patients, (subgroup A), and 20 older patients (subgroup B) (see Table I). The control group included ten patients under 40 years of age (subgroup C), and 18 patients over 40 (subgroup D) (see Table II).

A comparison of these subgroups showed the CST to be subnormal or borderline in three out of seven treated patients in subgroup $A$, and in nine of the 20 patients in subgroup B. In the untreated control patients the results of this test proved normal in all the C control subgroup, and pathologic or borderline in three of the 18 in D control subgroup.

On the other hand, normal EOG was seen in all subgroup $A$ patients, and subnormal
EOG in four of the 20 subgroup B subjects (Table I). In the control subgroups $(C, D)$ the EOG was normal in the entire $C$ subgroup, and subnormal in two of the 18 in D control subgroup (Table II).

The PVEP was subnormal in one patient of each of the A and B treated subgroups, and normal in all the subgroup $C$ and $D$ control patients.

\section{Discussion}

Plaquenil maculopathy, which is difficult to detect at the early, occasionally reversible stage, presents a challenge and a major medicolegal problem for ophthalmologists.

In screening for this maculopathy, one of the many tests used is EOG. However, its merits have recently been questioned, with the discovery of low values in untreated patients suffering from rheumatoid arthritis. ${ }^{15}$

The CST has been described as a sensitive test for several ocular conditions, including media haziness, ${ }^{16,17}$ maculopathies, ${ }^{11}$ glaucoma ${ }^{18}$ and diseases of the optic nerve..$^{19,20}$

Our study of 27 patients treated with hydroxychloroquine for long periods, ranging from one to nine years, showed that it was possible to detect abnormal macular function far more readily by screening these patients with the CST than with either the EOG or PVEP tests. These findings were highly significant as shown by $\chi$-square statistical analysis of the three tests, and in a comparison between the control and treated groups.

In the treated patients the CST was pathologic or suspect in $44.4 \%$ of the cases (12 out of 27), a significantly high percentage compared with the $14.8 \%$ (four out of 27) found in the EOG test $(\mathrm{p}<0.02)$, or the $7.4 \%$ (two out of 27) in the PVEP test $(\mathrm{p}<0: 01)$.

In the untreated control group, the frequency of pathologic or suspected CST was $10.7 \%$ (three out of 28), as compared with $44.4 \%$ in the treated group, the difference between the two groups revealing a high statistical significance $(\mathrm{p}<0.005)$.

On the other hand no significant difference in the frequency of pathological changes in the EOG $(\mathrm{p}<0.99)$ was found between the control group (7.1\%, two out of 28$)$ and the treated group (14.8\%).

Since no pathological changes were found 
in the two subnormal PVEP subjects in the treated group, the results of the test were not considered to be of any significance.

It should be emphasised that the abnormal results were detected in fundi of normal appearance.

The reason for the three positive CST cases detected in the control group (Table II) is not clear. In two nuclear sclerosis may be the underlying cause, or in all three just a poor performance. Senile changes in the pigment epithelial cells, such as the accumulation of lipofuscein deposits, might account for the two positive EOG results among the control group.

The findings were more striking on comparison of the treated and untreated subgroups. The EOG proved normal in all the treated A subgroup and control $C$ subgroup, with no difference between the two, whereas the CST was abnormal in $42.88 \%$ (three out of seven) of the treated A subgroup, but normal in the entire matching control $\mathrm{C}$ subgroup $(p<0.05)$, statistically very significant.

Although significant, the difference was less conspicuous when the older subgroups were considered, with the EOG subnormal in $20 \%$ (four out of 20) of the treated B subgroup, compared with $11.1 \%$ (two out of 18 ) in the control D subgroup $(\mathrm{p}<0.5)$. The CST was subnormal in $45 \%$ (nine out of 20 ) of the treated B subgroup, compared with $16.7 \%$ (three out of 18) in the control D subgroup $(\mathrm{p}<0.1)$.

It should be noted that improvement in the CST performance was evident in patients following discontinuation of medication, and in some of them the re-establishment of treatment caused reduction in the CST performance (data under evaluation, not included here), a fact which may lend support to the notion that the abnormal results are related to the antimalarial treatment.

Although the group studied was relatively small, the results support the fact that the CST is more effective and sensitive than the EOG in detecting macular hypofunction induced by antimalarial drugs. It should be borne in mind that the CST may show some false positive results with advancing age (Table II), although in patients under 40 its accuracy is much better. Using this test, macular abnor- mality was detected in $45 \%$ of the treated patients under 40 , but none in the control subgroup $(\mathrm{p}<0.05)$.

In conclusion, we consider that the CST is a suitable test for evaluation and follow up of chloroquine-induced maculopathy. It is sensitive, easy to perform and less time consuming than other tests. In addition, although not included in our study, it is less psychologically traumatic for the paediatric age group, which constitutes a considerable proportion of patients under antimalarial treatment.

We are grateful to Dr. Z. Awdeh for his help with the statistical analysis, and Ms. S. Dahan for her technical aid. Their assistance was invaluable.

\section{References}

${ }^{1}$ Scherbel A: Use of synthetic antimalarial drugs and other agents for rheumatoid arthritis: Historic and therapeutic perspective. Am J Ophthalmol 1983, 75: 1-4.

2 Paulus HE: An overview of benefit/risk of disease modifying treatment of rheumatoid arthritis as of today. Ann Rheum Dis 1982, 41 (supp.1): 26-9.

${ }^{3}$ Young $\mathrm{P}$ and Bronsan DW. Simple test for the early detection of retinopathy due to chloroquine and hydroxychloroquine. Abst.: Arthritis Rheum. 1963, 6: 798-9.

${ }^{4}$ Kearns TP and Hollenhorst PW. chloroquine retinopathy: Evaluation by fluorescein fundus angiography. Arch. Ophthalmol 1966, 76: 378-84.

${ }^{5}$ Okun E, Gouras P, Bernstein H, Von Sallman L. Chloroquine retinopathy: A report of eight cases with ERG and dark adaptation findings: Arch Ophthalmol 1963, 69: 59-71.

${ }^{6}$ Cruess AF, Schachat AP, Nicholl J, Ausburger JJ: Chloroquine retinopathy. Is Fluorescein angiopathy necessary? Ophthalmology 1985, 92: 1127-9.

${ }^{7}$ Easterbook M: The use of Amsler grids in early chloroquine retinopathy. Ophthalmology 1984, 91: $1368-72$.

${ }^{8}$ Arden GB, Friedman A, Kolb H: Anticipation of chloroquine retinopathy. Lancet 1962, 1: 1164-5.

${ }^{9}$ Gouras P and Gunkle RD: The EOG in chloroquine and other retinopathies. Arch Ophthalmol 1973, 70: 629-31.

${ }^{10}$ Van Lith GHM, Mark GTM, Winjnands H. Clinical importance of the electrooculogram with special reference to chloroquine retinopathy. Bibl. Ophthalmol. 1976, 85: 2-9.

${ }^{11}$ Sjöstrand J: Contrast sensitivity in macular disease using a small-field and a large-field TV system. Acta Ophthalmol 1979, 57: 832-45.

${ }^{12}$ Loshin DS and White J. Contrast sensitivity. The visual rehabilitation of the patient with macular degeneration. Arch Ophthalmol 1984, 102: 1303-6.

${ }^{13}$ Bass SJ, Sherman J, Bodis-Wollner I, Nath S. Visual 
evoked potentials in macular diseases. Invest. Ophthalmol. Vis. Sci. 1985, 26: 1071-4.

${ }^{14}$ Marcus M, Merin S, Wolf M, Feinsod M. Electrophysiologic tests in assessment of senile macular degeneration. Ann Ophthalmol. 1983, 15: 235-8.

15 Pinckers A and Broekhnyse RM. The EOG in rheumatoid arthritis. Acta Ophthalmol 1983, 61: 831-7.

${ }^{16}$ Hess RF and Garner LF. The effect of corneal oedema on visual function. Invest. Ophthalmol. Vis. Sci. 1977, 16: 5-13.
${ }^{17}$ Hess RF and Woo G: Vision through cataracts. Invest. Ophthalmol. Vis. Sci. 1978, 17: 428-35.

${ }^{18}$ Atkin A, Bodis-Wollner I, Wolkstein M, Moss A, Podos S. Abnormalities of central contrast sensitivity in glaucoma. Am. J. Ophthalmol 1979, 88: 205-11.

${ }^{19}$ Arden GB and Gucukoglu AG. Grating test of contrast sensitivity in patients with retrobulbar neuritis. Arch Ophthalmol 1978, 96: 1626-9.

${ }^{20}$ Regan D, Silver R, Murray JT: Visual acuity and contrast sensitivity in multiple sclerosis-hidden visual loss. An auxiliary diagnostic test. Brain 1977, 100: 563-79. 